\title{
Quantum stochastic description of collisions in a canonical Bose gas
}

\author{
Patrick Navez ${ }^{1}$ and Achilleas Lazarides ${ }^{2}$ \\ ${ }^{1}$ Institut für Theoretische Physik, TU Dresden, 01062 Dresden, Germany \\ ${ }^{2}$ Max Planck Institute for the Physics of Complex Systems, 01187 Dresden, Germany
}

(Dated: November 11, 2018)

\begin{abstract}
We derive a stochastic process that describes the kinetics of a one-dimensional Bose gas in a regime where three body collisions are important. In this situation the system becomes non integrable offering the possibility to investigate dissipative phenomena more simply compared to higher dimensional gases. Unlike the quantum Boltzmann equation describing the average momentum distribution, the stochastic approach allows a description of higher-order correlation functions in a canonical ensemble. As will be shown, this ensemble differs drastically from the grand canonical one. We illustrate the use of this method by determining the time evolution of the momentum mode particle number distribution and the static structure factor during the evaporative cooling process.
\end{abstract}

PACS numbers: 03.75.-b,03.75.Lm,05.45.Yv

The stochastic kinetic description of a quantum gas has been the subject of considerable activity [1-3]. The main idea is to describe the state in terms of a set of stochastic classical fields $\psi^{(i)}(r, t)(i=1, \ldots, I)$ associated to the bosonic quantum field $\psi(r, t)$ whose averages give access to the n-point correlation functions, possibly in the CN (canonical) ensemble. The determination of these quantities has been shown to be of relevance in recent interference experiments with cold atoms [4] where mode probability functions have triggered considerable interest [5, 6]. As discussed in [1], the main difficulty is to be able to derive an associated Fokker-Planck(FP)like equation for the probability in the $\mathrm{P}$ representation which can be recasted into a stochastic Langevin equation. Such a task cannot be achieved exactly since the resulting equation would be a non dissipative FP equation [1, 7-9]. Only the use of the positive $\mathrm{P}$ representation appears to be tractable [9]. Some approximations have to be made in a bid to obtain numerically solvable dissipative equations. For the general case of finite temperature, only a phenomenological approach is available in which the stochastic terms guarantee the approach towards thermodynamic equilibrium without the requirement of an additional cutoff at large momentum [3].

In this Letter, we present a microscopic derivation of a Langevin equation starting from the full many body Hamiltonian in which the stochasticity originates from collision processes. For simplicity, we study a one dimensional Bose gas subject to three-body interactions only (two-body interactions does not lead to effective thermalization in 1D). Such a system could be realized in cold atom experiments [10] and differs from the usual 1D exactly solvable Hamiltonian by the addition to the twobody quartic term of a sixtic interaction term resulting from virtual interaction with transverse degrees of freedom. Its study allows simpler access to the exploration of quantum collisions compared to the more involved higher dimensional systems [2, 11, 12], in particular to the study of their enhancement due to the Bose statistics. As an illustration, we consider the dynamics of evaporative cooling in the $\mathrm{CN}$ ensemble and show that the time evolution of quantities such as correlations or mode probability distribution differ strongly from the one obtained from the Boltzmann equation valid only in the grand canonical (GN) ensemble.

Defining the atom mass $m$, the scattering length $\alpha_{s}$, its $1 \mathrm{D}$ density $n$ and the transverse trap frequency $\omega_{r}$, we focus on the thermalization regime described in [10] characterized by a high transverse confinement $\hbar \omega_{r} \gg k_{B} T$, a weak correlation parameter $\gamma=2 \alpha_{s} m \omega_{r} / \hbar n \ll 1$ in order to violate integrability so that the three-body collision rate overcomes the two-body collision rate responsible for transverse mode excitations.

Restricting ourselves to a uniform gas and defining the spatial Fourier components of the field $\psi^{(i)}(r, t)=$ $\sum_{k} \exp (i k r) \alpha_{k}^{(i)}(t) / \sqrt{L}$, we shall show how to derive the following Langevin-type equation:

$$
d \alpha_{p}^{(i)}=\left(\Gamma_{p}^{i n}-\Gamma_{p}^{o u t}+i \omega_{p}^{\prime}\right) \alpha_{p}^{(i)} d t+\sqrt{2 \Gamma_{p}^{i n}} d \eta_{p}^{(i)}
$$

where $\omega_{p}^{\prime}$ is the kinetic and mean field energy of the atom, $\Gamma_{p}^{i n}(t)$ and $\Gamma_{p}^{o u t}(t)$ correspond to the ingoing and outgoing collision terms respectively. These are averaged functionals of the $\alpha_{p}^{(i)}(t)$ and are determined from Eq.(17]18). The noise $d \eta_{p}^{(i)}$ follows a Gaussian distribution with the only non trivial average $\left\langle d \eta_{p} d \eta_{p^{\prime}}^{*}\right\rangle=\delta_{p, p^{\prime}} d t$. In contrast to [2, 7], this equation includes both high and low energy modes in the stochastic process. The average is defined as $\langle A\rangle=\sum_{i=1}^{I} A^{(i)} / I$ for any set of realization $A^{(i)}$. It is done in the grand canonical (GC) ensemble and corresponds to the Monte-Carlo approximation of the integral over coherent state labeled by the $\alpha_{p}$ 's [3]. In other words, the expectation value of any observable functional of the creation annihilation operator $a_{p}^{\dagger}$ and $a_{p}$, is in the limit of a large set $I$, identical to an average over the stochastic variables $\alpha_{p}^{*}$ and $\alpha_{p}$.

We note the connection with the quantum Boltzmann equation. Multiplying (1) by its complex conjugate and taking the average over the ensemble, the stochastic equation is connected to the quantum Boltzmann equa- 
tion for the momentum distribution $\bar{n}_{k}=\left\langle n_{k}\right\rangle=\left\langle\left|\alpha_{k}\right|^{2}\right\rangle$ :

$$
\frac{d \bar{n}_{k}(t)}{d t}=2 \Gamma_{k}^{i n}\left(1+\bar{n}_{k}\right)-2 \Gamma_{k}^{o u t} \bar{n}_{k}
$$

This equation becomes closed if the $\Gamma_{k}^{\text {in }}$ and $\Gamma_{k}^{\text {out }}$ are functionals of $\bar{n}_{k}$. This is realized through the application of the Wick's decomposition theorem (which corresponds to the stosszahlansatz): defining the product $M_{n}\left(\left\{\alpha_{k}\right\}\right)=\alpha_{k_{1}}^{*} \ldots \alpha_{k_{n}}^{*} \alpha_{k_{1}^{\prime}} \ldots \alpha_{k_{n}^{\prime}}$, its average in the GC ensemble for a uniform gas is decomposed as:

$$
\left\langle M_{n}\left(\left\{\alpha_{k}\right\}\right)\right\rangle=\bar{n}_{k_{1}} \ldots \bar{n}_{k_{n}} \sum_{\left\{k_{j}\right\} \in P} \prod_{i} \delta_{k_{i}, k_{j}}
$$

where $P$ is the permutation ensemble. On the contrary, the stosszahlansatz is not necessary anymore in Eq.(1) and correlations can be taken into account.

Another advantage of the stochastic formalism is the possibility to relate the averages in the $\mathrm{CN}$ ensemble to the GC ones by means of the weight functions $W_{N}\left(\left\{\alpha_{k}\right\}\right)=e^{-\sum_{k}\left|\alpha_{k}\right|^{2}}\left(\sum_{k}\left|\alpha_{k}\right|^{2}\right)^{N} / N$ ! so that for $N$ atoms we obtain for any product [3] :

$$
\left\langle M_{n}\left(\left\{\alpha_{k}\right\}\right)\right\rangle_{N}=\frac{\left\langle W_{N-n}\left(\left\{\alpha_{k}\right\}\right) M_{n}\left(\left\{\alpha_{k}\right\}\right)\right\rangle}{\left\langle W_{N}\left(\left\{\alpha_{k}\right\}\right)\right\rangle}
$$

The weight function plays the role of a projection operator restricting the particle number to $N$. Probability distribution in the $\mathrm{CN}$ ensemble can also be determined for mode population. For example, defining the weight functions $W_{N, k \neq 0}=e^{-\sum_{k \neq 0}\left|\alpha_{k}\right|^{2}}\left(\sum_{k \neq 0}\left|\alpha_{k}\right|^{2}\right)^{N} / N$ ! and $W_{N, k=0}=e^{-\left|\alpha_{0}\right|^{2}}\left|\alpha_{0}\right|^{2 N} / N$ !, the probability distribution for the zero momentum mode reads:

$$
P_{N}\left(n_{0}\right)=\frac{\left\langle W_{N-n_{0}, k \neq 0}\left(\left\{\alpha_{k}\right\}\right) W_{n_{0}, k=0}\left(\left\{\alpha_{k}\right\}\right)\right\rangle}{\left\langle W_{N}\left(\left\{\alpha_{k}\right\}\right)\right\rangle}
$$

The determination of the stochastic equation is done as follows. We start from the ternary many body Hamiltonian [10]:

$$
H=\sum_{k} \omega_{k} c_{k}^{\dagger} c_{k}-\frac{g_{3}}{L^{2}} \sum_{\sum_{i} p_{i}=\sum_{i} q_{i}} c_{p_{1}}^{\dagger} c_{p_{2}}^{\dagger} c_{p_{3}}^{\dagger} c_{q_{1}} c_{q_{2}} c_{q_{3}}
$$

where $\omega_{k}=k^{2} / 2 m$ is the atom kinetic energy and $g_{3}=2 \ln (4 / 3) \hbar \omega_{r} \alpha_{s}^{2}$ is the ternary three body interactions. The suppression of the quartic interactions proportional to $g_{2}=\hbar \omega_{r} \alpha_{s}$ is valid in a region where the mean field interaction energy is negligible compared to the kinetic energy i.e. $g_{2} n \ll k_{B} T$ so that the phonon excitation energy are mostly particle-like [10]. Starting the density matrix $\rho(t)$, we obtain the reduced density matrix for the momentum mode $p$ by taking the trace over all other modes:

$$
\rho_{p}(t)=\operatorname{Tr}_{\backslash p}(\rho(t))
$$

The stochastic time evolution of the mode $p$ is derived by considering the other modes as a bath for this mode. For
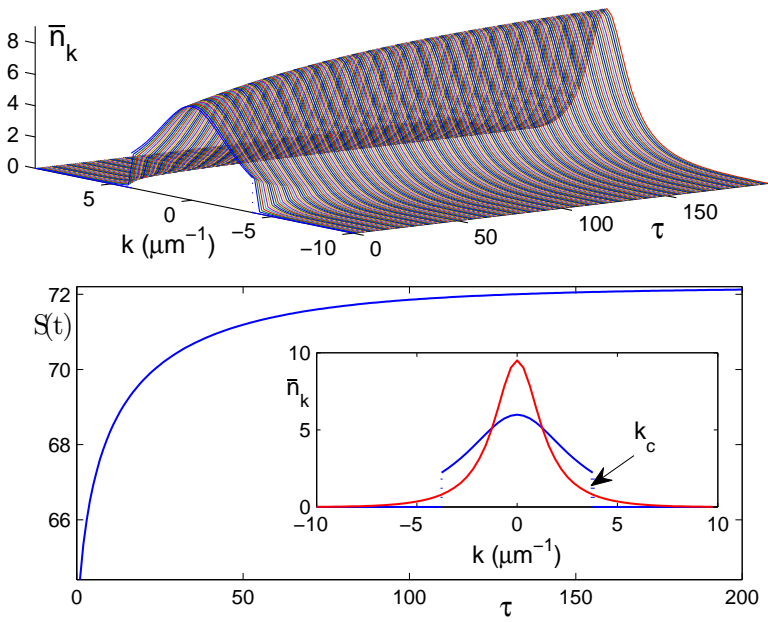

FIG. 1. Evolution of the momentum average distribution $n k$ vs. the reduced time $\tau$. The corresponding entropy is shown below and indicate how the distribution is close to equilibrium. Initial and final distributions are shown in blue and red respectively in the inset.

this purpose, we decompose the density matrix in terms of an uncorrelated contribution and a correlated one:

$$
\rho(t)=\prod_{p} \rho_{p}(t)+\delta \rho(t)
$$

The Hamiltonian can be decomposed into three terms:

$$
H=H_{1}+H_{2}+H_{3}
$$

where $n_{k}=a_{k}^{\dagger} a_{k}$ and

$$
\begin{gathered}
H_{1}=\omega_{p} n_{p}+\mathcal{O}(1 / L) \\
H_{2}=A_{p} n_{p}+C_{p}^{\dagger} a_{p}+a_{p}^{\dagger} C_{p}+\mathcal{O}(1 / L)
\end{gathered}
$$

and where $A_{p}, C_{p}$ and $H_{3}$ are operators describing the other modes. We omit terms of the order $\mathcal{O}(1 / L) \ll n$ which are negligible in the thermodynamic limit. Using the formalism in [8] to derive the master equation up to the second order in $g_{3}$, we obtain:

$$
\begin{aligned}
\frac{\partial \rho_{p}}{\partial t} & =-\frac{i}{\hbar}\left[H_{1}^{\prime}, \rho_{p}\right]+\Gamma_{p}^{\text {in }}(t)\left(2 a_{p}^{\dagger} \rho_{p} a_{p}-\left[a_{p} a_{p}^{\dagger}, \rho_{p}\right]_{+}\right) \\
& -\Gamma_{p}^{o u t}(t)\left(\left[a_{p}^{\dagger} a_{p}, \rho_{p}\right]_{+}-2 a_{p} \rho_{p} a_{p}^{\dagger}\right)
\end{aligned}
$$

where

$$
H_{1}^{\prime}=H_{1}+\operatorname{Tr}_{\backslash p}\left(\prod_{k \neq p} \rho_{k} H_{2}\right)=\omega_{p}^{\prime} n_{p}
$$

defining $\omega_{p}^{\prime}=\omega_{p}-3 ! g_{3} n^{2}$ and $n=\sum_{k}\left\langle n_{k}\right\rangle / L$ and where

$$
\begin{aligned}
\Gamma_{p}^{\text {in }}(t) & =\operatorname{Re} \frac{1}{\hbar^{2}} \int_{0}^{\infty} d t^{\prime}\left\langle C_{p}^{\dagger}\left(t^{\prime}\right) C_{p}\right\rangle \\
& =\operatorname{Re} \frac{1}{\hbar^{2}} \int_{0}^{\infty} d t^{\prime} \operatorname{Tr}\left(C_{p}^{\dagger}\left(t^{\prime}\right) C_{p} \prod_{k \neq p} \rho_{k}(t)\right) \\
\Gamma_{p}^{\text {out }}(t) & =\operatorname{Re} \frac{1}{\hbar^{2}} \int_{0}^{\infty} d t^{\prime}\left\langle C_{p}\left(t^{\prime}\right) C_{p}^{\dagger}\right\rangle
\end{aligned}
$$


Terms containing the operator $A_{p}$ do not contribute up to $g_{3}^{2}$ by symmetry between the in and the out terms. The only contribution comes from the operator $C_{p}$ which expressed in the interaction picture becomes:

$$
C_{p_{1}}(t)=\frac{3 g_{3}}{L^{2}} \sum_{\sum_{i} p_{i}=\sum_{i} q_{i}} e^{i \sum_{i}\left(\omega_{p_{i}}-\omega_{q_{i}}\right) t} c_{p_{2}}^{\dagger} c_{p_{3}}^{\dagger} c_{q_{1}} c_{q_{2}} c_{q_{3}}(16)
$$

The stochastic equation can be implemented numerically directly using Eq. (14)15) but in order to avoid the summation over too many momentum variables we make the random phase approximation. For a uniform gas, we can neglect off-diagonal contributions in the $p_{i}$ and $q_{i}$ since they induce a phase factor that appears to be random and thus cancels in the summation process. Thus, only diagonal components remain. Carrying out the integral over $t^{\prime}$, we obtain with the following expression:

$$
\begin{aligned}
\Gamma_{p}^{\text {in }}(t) & =(3 !)^{2} \pi\left(\frac{g_{3}}{\hbar L^{2}}\right)^{2} \sum_{\left\{p_{i}, q_{i}\right\}} \delta_{\sum_{i} p_{i}, \sum_{i} q_{i}} \delta\left(\sum_{i} \omega_{p_{i}}-\sum_{i} \omega_{q_{i}}\right)\left\langle n_{p_{2}} n_{p_{3}}\left(n_{q_{1}}+1\right)\left(n_{q_{2}}+1\right)\left(n_{q_{3}}+1\right)\right\rangle \\
\Gamma_{p}^{\text {out }}(t) & =(3 !)^{2} \pi\left(\frac{g_{3}}{\hbar L^{2}}\right)^{2} \sum_{\left\{p_{i}, q_{i}\right\}} \delta_{\sum_{i} p_{i}, \sum_{i} q_{i}} \delta\left(\sum_{i} \omega_{p_{i}}-\sum_{i} \omega_{q_{i}}\right)\left\langle n_{q_{1}} n_{q_{2}} n_{q_{3}}\left(n_{p_{2}}+1\right)\left(n_{p_{3}}+1\right)\right\rangle
\end{aligned}
$$

Note the Bose enhancement factor (terms $n_{p}+1$ ) that amplifies the collision process when the output modes are already populated. From this form we can deduce the FP equation associated to the master equation (12):

$$
\frac{\partial P_{p}(t)}{\partial t}=\left\{-i \omega_{p}^{\prime}\left(\alpha_{p} \frac{\partial}{\partial \alpha_{p}}-\alpha_{p}^{*} \frac{\partial}{\partial \alpha_{p}^{*}}\right)+\left(\Gamma_{p}^{o u t}(t)-\Gamma_{p}^{i n}(t)\right)\left(\frac{\partial}{\partial \alpha_{p}} \alpha_{p}+\frac{\partial}{\partial \alpha_{p}^{*}} \alpha_{p}^{*}\right)+2 \Gamma_{p}^{i n}(t) \frac{\partial}{\partial \alpha_{p}} \frac{\partial}{\partial \alpha_{p}^{*}}\right\} P_{p}(t)
$$

from which we deduce the Langevin equation Eq.(11). The solution of this equation is a Gaussian distribution $P_{p}(t)=\exp \left(-\left|\alpha_{p}\right|^{2} / \bar{n}_{p}(t)\right) / \bar{n}_{p}(t)$ with the normalization $\int d^{2} \alpha_{p} P_{p}(t) / \pi=1$ and where $\bar{n}_{p}(t)$ has to fulfill Eq.(2). In equilibrium in the GC ensemble, through the application of Eq.(3) on Eq.(1718), we recover the Bose-Einstein distribution $\bar{n}_{k}=1 /\left[\exp \left(\left(\omega_{p}-\mu\right) / k_{B} T\right)-1\right]$ as the stationary solution where the parameter $\mu$ defines the chemical potential.

For an illustration of all these considerations, we apply the stochastic method to the process of evaporative cooling. We take a gas of ${ }^{87} R b$ of $n=5.25 \mu m^{-1}$ confined in a box of size $L=20 \mu \mathrm{m}$ with $\omega_{r} / 2 \pi=6 \mathrm{kHz}$ and $\alpha_{s}=5.3 \mathrm{~nm}$. We choose the dimensionless time $\tau=10^{6} t / t^{*}$ where $1 / t^{*}=(3 !)^{3}\left(g_{3} / \hbar L\right)^{2} m / \pi \hbar$.

We start from an initial Bose gas at high temperature $T_{i}=29 n K$, we then remove the hottest atom with momentum higher than $k_{c}=4 \mu m^{-1}$ and study the relaxation process of this cut distribution towards an equilibrium one with a lower temperature $T_{f}=9 n K$ at a time estimated to $t=0.14 \mathrm{~s}(\tau=200)$. The time evolution of the GC distribution calculated from Boltzmann approach of Eq.(2) is shown in Fig.1 together with the entropy $S(t)=\sum_{p}\left(\bar{n}_{p}+1\right) \log \left(\bar{n}_{p}+1\right)-\bar{n}_{p} \log \left(\bar{n}_{p}\right)$ evolution that allows to monitor the speed at which the equilibrium state is reached. Its production can be shown to be always positive and stops at equilibrium [13].

The stochastic approach allows a more refined description in the GC ensemble. For instance, the atom probability distribution for the momentum mode is determined from the solution of Eq. (19) and corresponds to a Poisson

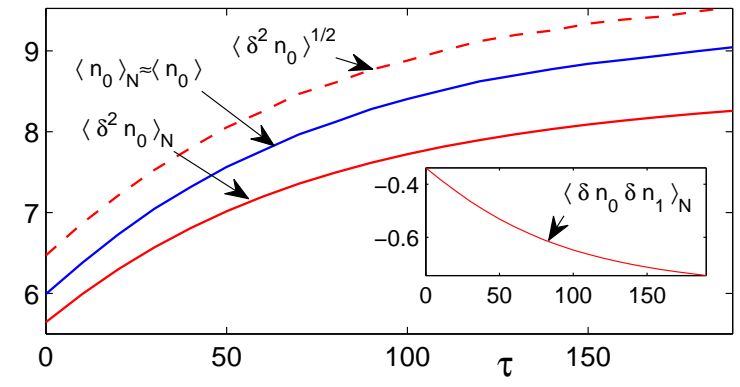

FIG. 2. Evolution of the $\mathrm{CN}$ and GC mean atom number in the zero mode and their fluctuations vs. the reduced time $\tau$. The negative correlation with the first excited mode is shown in the inset.

distribution:

$$
P\left(n_{p}\right)=\int \frac{d^{2} \alpha_{p}}{\pi} \frac{\left|\alpha_{p}\right|^{2 n_{p}}}{n_{p} !} e^{-\left|\alpha_{p}\right|^{2}} P_{q}=\frac{\bar{n}_{p}^{n_{p}}}{\left(\bar{n}_{p}+1\right)^{n_{p}+1}}(20)
$$

with the larger fluctuations $\left\langle\delta^{2} n_{0}\right\rangle_{G C}=\bar{n}_{0}\left(\bar{n}_{0}+\right.$ 1). Correlations between modes are non existent e.g. $\left\langle\delta n_{0} \delta n_{1}\right\rangle_{G C}=0$ where $n_{1}$ is the mode for the first excited state $k=2 \pi / L$.

The situation changes drastically in a canonical description as the fixed atom number restricts the possibility of fluctuations. Using the formulae (4) and (5) over a sample of $10^{6}$ variables for the stochastic process (11), if the average value remains practically unchanged, the fluctuations are significantly reduced as shown as in Fig.2. The process of evaporative cooling has the effect to increase these fluctuations together with the mean popu- 


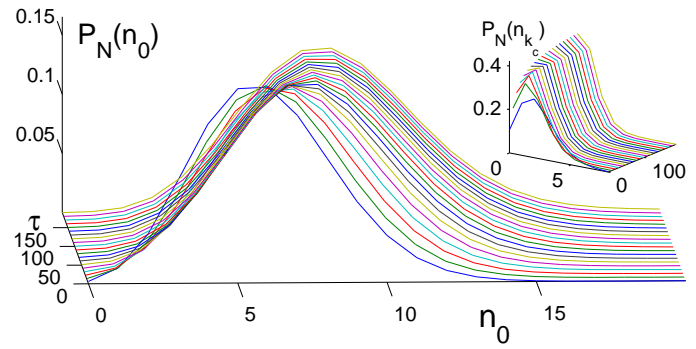

FIG. 3. Evolution of the $\mathrm{CN}$ atom probability distribution function in the zero mode vs. the reduced time $\tau$. For comparison, the distribution for the mode $k_{c}$ is represented in the inset.

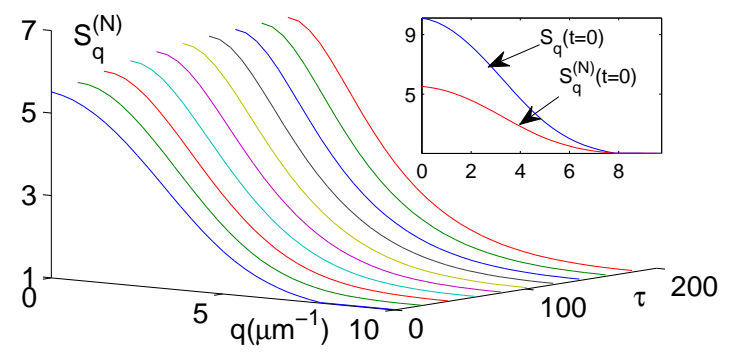

FIG. 4. Evolution of the static structure factor $S_{q}^{(N)}$ in the CN ensemble. For comparison, the corresponding GC function $S_{q}(t=0)$ is represented in the inset.

lation. The negative values of $\left\langle\delta n_{0} \delta n_{1}\right\rangle_{C N}$ is an evidence of correlations between modes in the $\mathrm{CN}$ ensemble.

The canonical probability distribution resembles a Gaussian one. Fig.3 shows how this distribution evolves with time and illustrates the benefit of using a stochastic approach for quantities that differ according ensembles. For the mode $k_{c}$, the distribution is initially peaked and becomes monotonic during the time evolution.

The static structure factor is defined in GC and CN ensembles respectively as [14]:

$$
S_{q}=\left\langle\delta \rho_{q} \delta \rho_{-q}\right\rangle / N \quad S_{q}^{(N)}=\left\langle\delta \rho_{q} \delta \rho_{-q}\right\rangle_{N} / N
$$

and corresponds to the density fluctuations $\rho_{q}=$ $\sum_{k} c_{k-q}^{\dagger} c_{k}$. These functions can be measured in Bragg spectroscopy experiments [15] and reach unity for large momentum $q$. In Fig 4, we determine the time evolution of these fluctuations during the evaporative cooling process. We notice that the presence of correlation in the $\mathrm{CN}$ ensemble reduces significantly the static structure factor.

In conclusion, we have reformulated kinetic theory in terms of a stochastic approach where the stochastic variables describe the various momentum modes of the gas. Through a specific weight average, the CN description becomes accessible and shows drastic differences in comparison with the GC one. The method has been used for a one-dimensional gas but is quite general and can be applied to more general cases of higher dimension, including higher order interaction terms or in presence of longitudinal trap confinement.

PN would like to thank S. Heller, H. Hauptmann, S. Krönke and W. Strunz for helpful discussions.
[1] M. J. Steel, M. K. Olsen, L. I. Plimak, P. D. Drummond, S. M. Tan, M. J. Collett, D. F. Walls, and R. Graham Phys. Rev. A 58, 48244835 (1998).

[2] Proukakis N.P. and Jackson B., J. Phys. B: At. Mol. Opt. Phys. 41203002 (2008); S. P. Cockburn1, A. Negretti, N. P. Proukakis, and C. Henkel, Phys. Rev. A 83, 043619 (2011).

[3] S. Heller and W.T. Strunz, J. Phys. B 42, 081001 (2009); S. Heller's thesis.

[4] S. Hofferberth et al., Nature Physics 4, 489 (2008)

[5] V. Gritsev, E. Altman, E. Demler, A. Polkovnikov, Nature Physics 2, 705 (October 2006).

[6] S.F Rath and W. Zwerger, Phys. Rev. A 82, 053622 (2010).

[7] H.T.C. Stoof, Phys. Rev. Lett. 78, 768 (1997).

[8] C. Gardiner, Quantum Noise (Springer Verlag, 1990).
[9] P. Deuar and P.D. Drummond, Phys. Rev. Lett. 98, 120402 (2007); P. Deuar and P.D. Drummond, Phys. Rev. A 66, 033812 (2002); P.D. Drummond, Phys. Rev. Lett. 92, 040405 (2004).

[10] I. E. Mazets, J. Schmiedmayer, New J. Phys. 12, 055023 (2010).

[11] A. Griffin, T. Nikuni, E. Zaremba, Bose-condensed gases at finite temperatures (Cambridge University Press, 2009).

[12] Patrick Navez, Physica A 356, 241-278 (2005).

[13] R. Balescu, Equilibrium and nonequilibrium statistical mechanics (New York, Wiley-Interscience, 1975).

[14] L. Pitaevski, S. Stringari, Bose-Einstein condensation (Clarendon Press, 2003).

[15] Stamper-Kurn et al., Phys. Rev. Lett. 83, 2876 (1999). 